\title{
Mesure de l'humidité des sols par une méthode capacitive : analyse des facteurs influençant la mesure
}

\author{
JC Gaudu 1 JM Mathieu 2, JC Fumanal 3, L Bruckler 1*, \\ A Chanzy 1, P Bertuzzi 1, P Stengel 1, R Guennelon 4 \\ ${ }_{1}^{1}$ NRA, Unité de science du sol, BP 91, domaine St-Paul, 84143 Montfavet Cedex; \\ 2 IUT, département de génie électrique et informatique industriels; \\ ${ }_{3}$ IUT, département de mesures physiques, rue des Géraniums, 13337 Marseille Cedex 14; \\ ${ }^{4}$ INRA, Unité de science du sol, route de Saint-Cyr, 78026 Versailles Cedex, France
}

(Reçu le 31 juillet 1992; accepté le 13 octobre 1992)

\begin{abstract}
Résumé - On teste en laboratoire et in situ les performances d'un capteur capacitif destiné à la mesure de l'humidité du sol. En laboratoire, on montre que les dérives thermiques propres à l'appareillage sont négligeables, que la mesure de permittivité diélectrique relative est dépendante de la qualité du contact sol-électrodes, et que le volume de mesure a des dimensions de l'ordre de quelques $\mathrm{cm}^{3}$. Les relations linéaires permittivité relative-teneur en eau volumique sont dépendantes de la texture, de la structure (agrégats de $2-3 \mathrm{~mm}$ et $4 \mathrm{~mm}$ disposés autour des électrodes ou structure continue), de la température (la variation de la permittivité relative en fonction de la température peut atteindre 0,20 par ${ }^{\circ} \mathrm{C}$ dans la gamme de 0 à $45^{\circ} \mathrm{C}$ ), de la salinité (la mesure de permittivité diélectrique relative est peu sensible à la concentration saline jusqu'à des valeurs de conductance électrique de l'ordre de 2 à $3 \mathrm{mS}$ ). In situ, deux expérimentations sont réalisées, l'une sur un sol nu présentant un état structural homogène, l'autre sur un sol couvert (soja) présentant un état structural plus hétérogène. Pour chaque expérimentation, on réalise pendant plusieurs jours (21 et 41 j respectivement) des mesures simultanées de permittivité diélectrique, de température, de conductance électrique, et des contrôles de teneur en eau dans les couches superficielles du sol $(0$ à $20 \mathrm{~cm})$. Les relations d'étalonnage permittivité-humidité sont généralement linéaires et variables d'un capteur à l'autre. Cette variabilité est probablement due à la variabilité spatiale de la teneur en eau du sol, de la structure à proximité immédiate du capteur, eV ou du contact sol-électrodes. Par ailleurs, les effets thermiques sur la permittivité mesurée sont comparables à ceux obtenus en laboratoire, et la gamme de conductances électriques rencontrées in situ semble compatible avec celle ne présentant pas d'influence notable sur la mesure de permittivité et estimée en laboratoire.
\end{abstract}

permittivité diélectrique / humidité / texture / structure du sol / salinité / température

Summary - Soil moisture measurement using a capacitive probe. The performance of a capacitive probe for soil moisture measurement was studied under laboratory and field conditions. The probe (21-mm ED cylinder) was composed of 2 electrodes connected to an electronic oscillator $(38 \mathrm{MHz})$ located in the probe. Four soils were used to analyse the 'dielectric permittivity-volumetric water content' relationships under laboratory or field conditions. The sensitivity of the probe including its electronic components in relation to the variations of the temperature $\left(0-45^{\circ} \mathrm{C}\right)$ was very low (< 0.5 permittivity unit). Permittivity measurements were dependent on the quality of the soil-electrodes contact, and on the soil structure near the electrodes. The order of magnitude of the volume of influence was a few cubic centimeters, and the area of the soil most affected by the permissibility measurements was located in the vicinity of the electrodes. The 'dielectric permittivity-volumetric water content' relationships were linear $(r>0.99)$, and dependent on soil texture and structure. Moreover, soil temperature influenced the measurements since increases of the order of magnitude of 0.20 permittivity unit per ${ }^{\circ} \mathrm{C}$ could be obsenved. Permittivity measurements were not sensitive to the concentration of the solution when the electric conductance was $<2-3 \mathrm{~ms}$. Two field experiments were performed using 2 different soil textures. The first soil ( $C=10.5 \%, L=50.6 \%, S=38.8 \%)$ was a bare soil, and showed a quite homogeneous soil structure, the second $(C=27.2 \%, L=61.7 \%, S=11.1 \%)$ was cultivated with soybean, and showed a more heterogeneous soil structure. For each experiment field, simultaneous measurements ( 1 or 2 times per d) of relative dielectric permittivity, soil temperature and gravimetric soil water content were made in the vicinity of

* Correspondance et tirés à part 
the probes (over a 21 and $41 \mathrm{~d}$ period, for the 1st and 2nd experiment, respectively). The variation of the relative dielectric permittivity versus time was very similar to the variation of the measurement soil water content. The calibration relationships between the volumetric water content and the relative dielectric permittivity were linear for each capacitive probe. The regression lines for the different capacitive probes exhibited variations between probes, probably due to the spatial variability of the water content, soil structure and/or soil-electrode contact for each sensor. The effects of the soil temperature on the relative dielectric permittivity measurements were similar to the previous results obtained under laboratory conditions, and effects of electrical conductance appeared negligible under our conditions.

\section{dielectric permittivity / water content / texture / soil structure / salinity / temperature}

\section{INTRODUCTION}

Il n'existe pas à l'heure actuelle de méthode de mesure unique de la teneur en eau, universelle et applicable à toutes les situations, mais au contraire, une gamme assez vaste de méthodes souvent complémentaires les unes par rapport aux autres (Baron et Tran Ngoc Lan, 1977; Schmugge et al, 1980). Parmi ces techniques, celles basées sur les relations entre l'humidité et les propriétés diélectriques des sols se développent régulièrement. On peut citer la rétrodiffusion des micro-ondes actives (Ulaby, 1974), la réflectrométrie temporelle (Topp et al, 1982) (time domain reflectrometry, TDR) et la méthode capacitive.

La méthode capacitive (Paquet, 1965; Tran Ngoc Lan et al, 1970; Paquet, 1971; Ambrosino, 1972; Tran Ngoc Lan et al, 1972; Manière et al, 1974; Tran Ngoc Lan et Jallet, 1974; Hamid et Mostowy, 1976; Wobschall, 1978; Kuraz, 1981; Saxena et Tayal, 1981; Bell et al, 1987; Dean et al, 1987; Campbell, 1990) est basée sur la mesure de la permittivité diélectrique relative du sol à l'aide d'un pont capacitif ou d'un circuit électrique résonant, cette permittivité diélectrique relative étant elle-même très dépendante de la teneur en eau du sol dans la gamme de fréquence choisie, de 1 à $100 \mathrm{MHz}$ en général. La partie réelle de la permittivité diélectrique relative de l'eau libre est égale à 80 , celle des constituants solides du sol est comprise entre 2 et 7 environ, alors que celle de l'air est égale à 1. Le sol étant un système triphasique, composé d'une matrice solide présentant des pores occupés en proportion variable par des gaz et la solution du sol, son comportement diélectrique global dépendra de la permittivité diélectrique relative, de l'arrangement, et de la concentration volumique de chacun de ses constituants élémentaires, en particulier de l'eau. Cette méthode, déjà ancienne, a fait l'objet de nombreux travaux depuis 2 à 3 décennies. Ces travaux s'expliquent d'une part, par la variété des différents systèmes de mesure proposés, qui se différencient par la fréquence de mesure, la technologie électronique utilisée, la technique d'implantation du capteur en liaison avec sa géométrie, le degré d'automatisation de la chaîne de mesure, et d'autre part, par l'intérêt de ces méthodes, du point de vue des possibilités d'application. En effet, la méthode capacitive s'applique à de nombreux types de matériaux (bétons, platres, sols naturels, etc), et concerne en général de faibles volumes de mesure, ce qui permet une description fine des milieux stratifiés par exemple. Elle est automatisable, et permet donc d'obtenir la continuité des mesures dans le temps, et elle ne pose pas de problème relatif à la sécurité comme les méthodes neutroniques. En revanche, il existe un grand nombre de facteurs autres que l'humidité influençant la mesure. Parmi ceux-ci, on peut retenir la texture (Paquet, 1965; Tran Ngoc Lan et al, 1970; Tran Ngoc Lan et al, 1972; Manière et al, 1974; Kuraz, 1981; Bell et al, 1987; Campbell, 1990), la structure (Tran Ngoc Lan et al, 1970; Tran Ngoc Lan et al, 1972; Manière et al, 1974; Kuraz, 1981), la température (Kuraz , 1981; Campbell, 1990), la salinité (Tran Ngoc Lan et al, 1970; Kuraz, 1981), le contact sol-capteur (Dean et al, 1987). Dans cette diversité, les études présentant une analyse assez complète des propriétés des capteurs utilisés et des facteurs d'influence de la mesure sont en général peu nombreuses (Tran Ngoc Lan et al, 1970; Bell èt al, 1987; Dean et al, 1987).

Cet article se propose d'évaluer en laboratoire et in situ les performances du prototype d'un capteur capacitif conçu initialement par l'Institut universitaire de technologie de Marseille et l'Institut national de la recherche agronomique, en vue de sa commercialisation (Nardeux, St-Avertin, France). Les principaux choix techniques ayant présidé à la conception de ce capteur sont les suivants :

- automatisation de la chaîne de mesure pour des suivis à long terme de la teneur en eau du sol en laboratoire ou in situ, 
- volume de mesure permettant une description la plus fine possible (quelques $\mathrm{cm}$ ) des horizons superficiels du sol ou des interfaces entre horizons;

- minimisation des perturbations d'origine thermique ou liées à la concentration saline de la solution du sol;

- implantation verticale du capteur après forage préalable d'un accès, permettant une mise en place peu perturbatrice et l'accès facile en profondeur.

\section{MATÉRIEL ET MÉTHODES}

\section{Principe de mesure}

Un diélectrique est un corps qui peut emmagasiner de l'énergie électrostatique. Lorsqu'il est placé entre les armatures d'un condensateur, un diélectrique parfait est susceptible de maintenir constante une tension aux bornes de ce dernier sans autre apport d'énergie, cette propriété résultant de phénomènes de polarisation des porteurs de charges liés. Dans le cas d'un diélectrique non parfait comme le sol, il existe des déplacements de porteurs de charges libres, donnant lieu à des phénomènes de conduction électrique et à des pertes d'énergie par effet Joule, liées à l'existence de forces de frottement (Paquet, 1965; Tran Ngoc Lan et al, 1970; Paquet, 1971). Lorsqu'un champ alternatif est appliqué aux bornes du condensateur, la conduction électrique et les frottements sont ainsi à l'origine d'un déphasage retard de la polarisation par rapport au champ appliqué. Pour traduire ces phénomènes, on caractérise le milieu par sa permittivité diélectrique relative, pour laquelle on utilise alors une notation complexe :

$$
\varepsilon=\varepsilon^{\prime}-\mathbf{j} \varepsilon^{\prime \prime}
$$

où $\varepsilon$ : permittivité diélectrique relative (sans dimension); $\varepsilon^{\prime}$ : composante réelle de la permittivité diélectrique relative (sans dimension); $\varepsilon^{\prime \prime}$ : composante imaginaire de la permittivité diélectrique relative (sans dimension).

D'un point de vue physique, la partie réelle de la permittivité diélectrique relative traduit l'aptitude du diélectrique à se polariser, la partie imaginaire correspondant aux phénomènes de pertes diélectriques responsables du déphasage entre le champ appliqué et la polarisation induite. Le principe de mesure consiste, après introduction de deux électrodes dans le sol, à considérer du point de vue électrique le système ainsi constitué (sol et électrodes introduites) comme un condensateur imparfait avec pertes (fig 1), constitué d'une capacité parfaite $(\gamma)$ et d'une résistance disposée en parallèle $(\rho)$. Le condensateur imparfait ainsi constitué est alors relié à un circuit électrique oscillant situé dans le corps du capteur (fig 1), dont on mesure la fré-

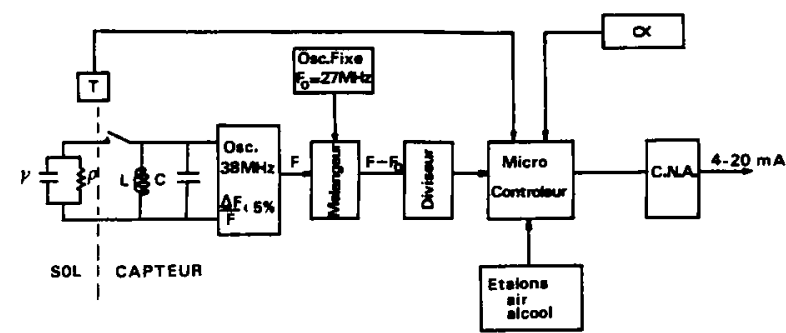

Fig 1. Schéma décrivant le principe de mesure de la chaîne électronique de mesure du capteur capacitif.

quence de résonance correspondant à la valeur de la capacité introduite. Cette capacité est directement reliée à la permittivité diélectrique relative du sol, par la relation suivante :

$$
\gamma / \gamma_{0}=\varepsilon^{\prime}
$$

où $\varepsilon^{\prime}$ : partie réelle de la permittivité diélectrique relative (sans dimension); $\gamma$ : valeur de la capacité électrique $(F) ; \gamma_{0}$ : valeur de la capacité électrique à vide (F),

alors que la résistance électrique globale du sol, assimilée à une résistance disposée en parallèle, est reliée à la partie imaginaire de la permittivité diélectrique relative du sol par la relation :

$$
\rho=1 / \varepsilon^{\prime \prime} \gamma_{0} \omega
$$

où $\rho$ : résistance de fuite disposée en parallèle $(\Omega)$; $\varepsilon^{\prime \prime}$ : partie imaginaire de la permittivité diélectrique relative (sans dimension); $\gamma_{0}$ : capacité électrique à vide (F); $\omega$ : pulsation ( rad s $^{-1}$ ).

Dans le cas du capteur étudié, la fréquence centrale du circuit oscillant est de $38 \mathrm{MHz}$. La conversion des valeurs de fréquence de résonance mesurées en valeurs de permittivité relative est obtenue par un étalonnage avec des milieux de permittivité diélectrique relative connue (1 pour l'air, et 25 pour l'alcool pur), la linéarité de cette relation étant vérifiée sur des milieux électriques de référence (Fumanal et al, 1989).

\section{Présentation du capteur capacitif}

La partie active du capteur (circuit électrique miniaturisé) est placée dans un cylindre de $21 \mathrm{~mm}$ de diamètre et de $135 \mathrm{~mm}$ de longueur, comportant les électrodes de mesure à son extrémité. Contrairement à des géométries d'électrodes rencontrées sur les dispositifs existants (disposition monoaxiale des électrodes pour laquelle 2 électrodes annulaires sont disposées sur le corps cylindrique du capteur, ou disposition pluriaxiale des électrodes à l'extrémité du corps du capteur), le capteur étudié comporte deux électrodes monoaxiales, une électrode annulaire de $10 \mathrm{~mm}$ de hauteur et de $21 \mathrm{~mm}$ de diamètre, et une 
électrode en pointe de $2 \mathrm{~mm}$ de diamètre et de $27 \mathrm{~mm}$ de longueur disposée à l'extrémité du corps cylindrique de la sonde. Cette disposition permet une implantation relativement facile du capteur, tout en permettant d'explorer un volume de sol peu perturbé plus important que celui obtenu dans le cas de réalisations antérieures. L'ensemble du dispositif est prévu pour fonctionner dans une plage de température comprise entre 0 et $45^{\circ} \mathrm{C}$, sans que des dérives thermiques liées aux variations de température des composants du capteur interviennent. Pour cela, un dispositif de double mesure de fréquence est mis en œuvre (fig 1), puisque le capteur est doté d'un relais activé toutes les 2 min permettant d'isoler un circuit interne au corps du capteur des électrodes implantées dans le sol : 2 mesures de fréquence sont alors effectuées, l'une prenant en compte la capacité constituée par le sol (relais fermé), l'autre prenant en compte uniquement le circuit électrique interne au capteur (relais ouvert). La différence de fréquence ainsi mesurée est alors uniquement fonction de la valeur constituée par la capacité constituée par le système sol-electrodes, et ne prend donc plus en compte des éléments capacitifs et inductifs propres au capteur, et sujets à des dérives thermiques ou à des vieillissements de composants électroniques.

La permittivité diélectrique relative des sols étant fonction de leur température (Hoekstra and Delaney, 1974; Campbell, 1990), le capteur dispose d'un dispositif permettant de convertir les mesures de permittivité relative obtenues à une température quelconque, en valeurs de permittivité relative normalisées à température constante $\left(20^{\circ} \mathrm{C}\right.$ par exemple). Cette procédure est rendue possible par l'incorporation d'un capteur de température interne au capteur (fig 2) couplé à un programme de calcul, et en supposant constante la variation de la permittivité relative en fonction de la température sur toute la gamme de variation de teneur en eau du sol.

Pour limiter les effets liés à la concentration saline de la solution du sol, le capteur comprend un dispositif électronique de compensation de l'influence de la conductivité électrique sur la mesure de permittivité relative (Fumanal et al, 1989), ce dispositif étant théoriquement opérationnel dans une gamme de conductance allant jusqu'à $5 \mathrm{mS}$.

\section{Expérimentations en laboratoire}

\section{Réponse du circuit électrique aux variations thermiques}

Deux expériences sont réalisées pour étudier la réponse du circuit électrique aux variations de température qu'il subit.

Dans une première expérimentation, le capteur est placé dans l'air à l'intérieur d'une chambre climatique. L'ensemble constitué par l'air et le capteur est alors soumis à des équilibres thermiques successifs dans

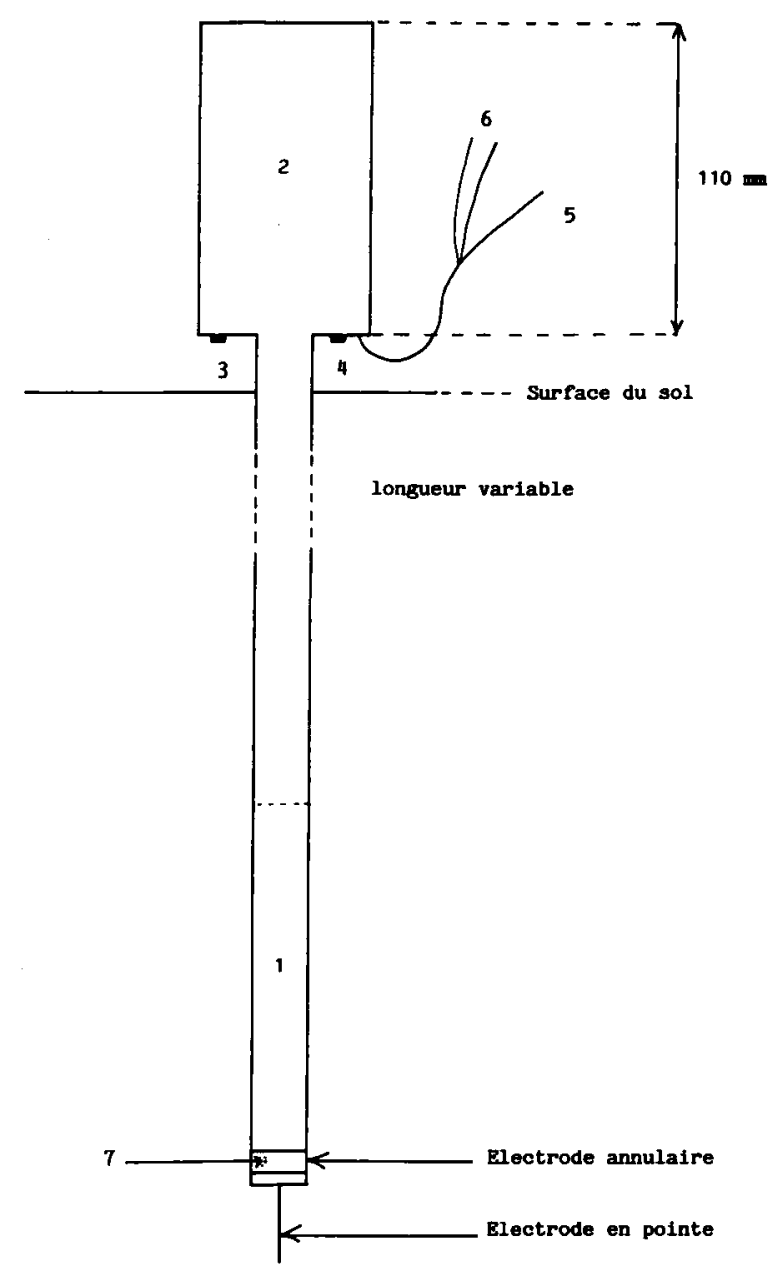

Fig 2. Géométrie, organisation des composants électroniques du capteur capacitif, et mise en place in situ. 1) Oscillateur $38 \mathrm{MHz}$, relais, amplificateur de température, mélangeur, oscillateur $27 \mathrm{MHz}$, diviseur; 2) convertisseur alimentation, convertisseur courant, microcontrôleur, programme; 3 ) roue codeuse 16 positions (réglage du coefficient de correction de température); 4) roue codeuse 3 positions (préétalonnage air-alcool); 5) sortie courant 4-20 mA; 6) alimentation 10-30 V; 7) capteur de température.

une gamme de température de 0 à $40^{\circ} \mathrm{C}$. La permittivité diélectrique relative de l'air étant assimilable à celle du vide et ne variant pas avec la température, les variations de permittivité relative fournies par le capteur sont alors une évaluation directe de l'importance des perturbations d'origine thermique sur la réponse du capteur. Dans une seconde expérimentation, on porte un bain d'alcool à des températures successives dans une gamme de 5 à $45^{\circ} \mathrm{C}$. À chaque température, deux mesures de permittivité relative sont effectuées :

- la première mesure est relevée instantanément après introduction du capteur capacitif dans l'alcool, lorsque ce capteur est encore à une température initiale voisine de $20^{\circ} \mathrm{C}$, quelle que soit la température de l'alcool; 
- la seconde est effectuée lorsque le capteur capacitif s'est mis en équilibre thermique avec le bain d'alcool.

La différence obtenue entre la première et la deuxième mesure n'est alors due qu'au changement de température du capteur capacitif entre ces deux mesures successives.

\section{Description de la géométrie du volume de mesure}

Pour caractériser le volume concerné par la mesure, on introduit le capteur dans un bain d'alcool (milieu homogène, permittivité diélectrique relative égale à 25 , et du même ordre de grandeur que celle d'un sol humide) contenu dans un récipient de grande dimension, afin de supprimer les effets de bord $(L=0,5 \mathrm{~m} ; \mathrm{l}=0,15 \mathrm{~m}$; $h=0,35 \mathrm{~m}$ ). La présence d'un interface air-alcool permet, par enfoncement progressif du capteur, de délimiter expérimentalement le volume de mesure dans les conditions de l'expérience. Lorsque la distance comprise entre l'interface air-alcool et l'extrémité de l'électrode en pointe augmente, la permittivité relative mesurée augmente également pour tendre vers celle de l'alcool. On établit alors une relation expérimentale entre le complément à 1 de l'erreur relative sur la permittivité relative de l'alcool $\left[1-\left(\Delta \varepsilon^{\prime} / \varepsilon^{\prime}\right)\right]$ et la cote d'enfoncement du capteur dans le liquide.

\section{Effet du contact sol-électrodes}

Une évaluation des risques générés par des phénomènes de retrait du sol autour des électrodes et du corps du capteur, ou d'une façon plus générale, par un mauvais contact sol-électrodes a été tentée en disposant de la paraffine $\left(\varepsilon^{\prime}=2,7\right)$ autour des électrodes et du corps du capteur pour simuler l'effet d'une lame d'air continue $\left(\varepsilon^{\prime}=1\right)$. Pour cela, une couche de paraffine d'épaisseur croissante est déposée autour du capteur par trempages successifs dans de la paraffine liquide. Malheureusement, une répartition uniforme de la paraffine sur le capteur n'a pu être obtenue à cause de l'écoulement de la paraffine liquide sur les électrodes. Pour évaluer malgré tout ces effets liés au contact solcapteur, nous avons changé la géométrie du capteur pour cette expérience en remplaçant l'électrode en pointe par un embout conique comportant une électrode annulaire. Pour cette géométrie, l'uniformité de l'épaisseur de la couche de paraffine sur la sonde est satisfaisante, et une mesure de permittivité relative dans un bain d'alcool est réalisée après chaque nouveau dépôt et séchage de paraffine sur le capteur (épaisseurs de paraffine allant de $0,4 \mathrm{~mm}$ à $4,6 \mathrm{~mm}$ ).

\section{Relations d'étalonnage permittivité relative-teneur en eau volumique}

\section{Choix des matériaux}

Quatre types de matériaux (teneurs en argile de $0 \%$ à $27,2 \%$ ) ont été retenus lors de l'étude du capteur en la- boratoire, afin de couvrir une gamme de variation suffisante (tableau I). Trois matériaux ont été prélevés in situ dans l'horizon $0-20 \mathrm{~cm}$, le quatrième étant constitué d'un sable fin pur.

\section{Préparation des échantillons}

Les échantillons de sol sont placés dans un moule de $15 \mathrm{~cm}$ de diamètre et de $6 \mathrm{~cm}$ de hauteur. Le capteur capacitif est placé dans un plan horizontal perpendiculaire à l'axe du moule, et à mi-hauteur de ce dernier. L'ensemble du dispositif ainsi constitué est placé dans un autre volume de plus grande dimension $(\mathrm{L}=$ $0,30 \mathrm{~m} ; \mathrm{l}=0,30, \mathrm{~h}=0,40 \mathrm{~m}$ ) et rempli de sol humide. Mise à part l'expérimentation visant à quantifier l'effet lié à la structure, les échantillons de sol sont préparés selon un protocole fixé. Pour les échantillons sableux, le remplissage du moule est effectué sous eau pour assurer un arrangement homogène des particules élémentaires. Pour les échantillons limoneux et limono-argileux, la structure initiale du matériau est obtenue par malaxage d'une pâte ayant une liquidité satisfaisante pour le remplissage du moule. L'échantillon évolue ensuite par pas successifs d'un état initial saturé vers un état final sec proche d'un arrangement textural des particules. Les variations de teneur en eau d'un état à l'autre sont obtenues soit par évaporation à partir de la face supérieure du moule, soit par succion à partir de la face inférieure. Après l'obtention de la teneur en eau moyenne souhaitée, le moule est fermé, et une période de redistribution de l'eau dans l'échantillon est alors imposée pour obtenir l'homogénéité souhaitée. Un critère quantitatif d'homogénéité, aux effets possibles d'hystérèse près entre le haut et le bas du cylindre, est obtenu à partir de 2 tensiomètres disposés horizontalement à $10 \mathrm{~mm}$ de chaque face du moule (face supérieure et face inférieure), dans une position diamétralement opposée. Au-delà de la gamme tensiométrique, des périodes de redistribution pouvant atteindre 15 à $20 \mathrm{j}$ sont déterminées sur un critère de stabilisation des mesures de permittivité relative au cours du temps. Les teneurs en eau

Tableau I. Composition granulométrique des matériaux utilisés en laboratoire (S31, Flassan, Marçon, Poirson) et in situ (Poirson, Collias).

Fraction

granulo- S31 Flassan Marçon Poirson Collias métrique

(\%)

$\begin{array}{lrrrrr}\text { Argile } & 0 & 7,9 & 14,4 & 27,2 & 10,5 \\ \begin{array}{l}\text { Limon fin } \\ \text { Limon }\end{array} & 0 & 3,4 & 31,0 & 41,7 & 14,5 \\ \text { grossier } & 0 & 2,5 & 41,2 & 20,0 & 36,2 \\ \begin{array}{l}\text { Sable fin } \\ \text { Sable }\end{array} & 100 & 41,7 & 9,9 & 7,1 & 15,7 \\ \text { grossier } & 0 & 44,5 & 3,5 & 4,0 & 23,1\end{array}$


massiques de l'échantillon de sol sont directement obtenues par pesée, et les masses volumiques par mesure du volume de sol et de la masse de sol sec. Dans le cas où l'échantillon présente des phénomènes de retrait au cours du séchage progressif, celui-ci est pris en compte pour le calcul de la masse volumique, en utilisant des mesures des dimensions de l'échantillon. Les différentes mesures, exception faite de l'éxpérimentation relative aux effets de la température du sol, ont été obtenues à une température de $25^{\circ} \mathrm{C}$.

\section{Effets de la texture}

L'expérimentation est conduite en utilisant 3 matériaux, présentés dans le tableau I (S31, Flassan, Marçon). Pour chaque cas, le moule est rempli selon les conditions standard exposées précédemment, et on suit une cinétique de dessèchement de l'échantillon la plus complète possible, afin d'explorer un domaine le plus large possible de teneur en eau.

\section{Effets de la structure}

L'étude n'a été réalisée que pour un seul matériau (Marçon), préparé selon 2 modalités différentes. La première modalité déjà décrite correspond à une structure continue (masse volumique moyenne de $1520 \mathrm{~kg} \mathrm{~m}^{-3}$ ). Pour la seconde modalité, on prépare des agrégats de sol tamisés (soit $2-3 \mathrm{~mm}$, soit $4 \mathrm{~mm}$ ) que l'on humecte par vaporisations successives jusqu'à obtention de la teneur en eau moyenne souhaitée. Pour chaque valeur d'humidité choisie, les agrégats sont placés manuellement autour du capteur capacitif dans le moule. Selon les teneurs en eau visées, les masses volumiques à létat sec sont comprises dans la gamme $\left(1060-1140 \mathrm{~kg} \mathrm{~m}^{-3}\right)$.

\section{Effets de la température}

L'étude a été conduite sur un seul matériau (Poirson), préparé selon les conditions standard et soumis à un dessèchement par paliers successifs dans une gamme de teneur en eau volumique allant de 0,536 $\mathrm{m}^{3} \mathrm{~m}^{-3}$ à $0,120 \mathrm{~m}^{3} \mathrm{~m}^{-3}$. À chaque palier, après la phase de redistribution, l'ensemble du dispositif expérimental est soumis à un essai thermique en armoire climatique, dans une gamme de température allant de 0 à $45^{\circ} \mathrm{C}$. Pour une teneur en eau donnée et une température donnée, les mesures de permittivité diélectrique relative sont effectuées après une durée d'équilibre thermique de $8 \mathrm{~h}$ dans l'enceinte climatique. Le milieu d'extension autour du moule est protégé de l'évaporation par un film en matière plastique durant la phase de mise en équilibre thermique.

\section{Effets de la salinité}

Cette expérimentation est destinée à évaluer les effets de la salinité de la solution du sol sur la réponse du capteur. La sonde capacitive est placée dans un sable pur inactif sur le plan physico-chimique luimême saturé soit par de l'eau distillée, soit par une solution de nitrate de potassium de concentration croissante (normalité de $110^{-5}$ à $310^{-2}$ ). Pour chaque concentration choisie, une mesure de permittivité relative est effectuée, et 5 mesures de permittivité relative sont également effectuées dans le sable saturé avec de l'eau distillée.

\section{Expérimentations in situ}

\section{Expérience 1}

Cet essai se déroule pendant $21 \mathrm{j}$ sur une cuve de sol enterrée, située dans le centre INRA de Montfavet (France). La cuve expérimentale ( $\mathrm{sol}$ nul) a une surface de $11,6 \mathrm{~m}^{2}(3,4 \times 3,4 \mathrm{~m})$, et une profondeur de $2 \mathrm{~m}$. Cette cuve est remplie d'un sol loessique provenant de Collias (Gard, France), de texture limono-sableuse (tableau I). Le remplissage de la cuve, effectué il y a plusieurs années sous eau, permet d'obtenir un matériau de structure homogène, relativement compact, et n'ayant pas été perturbé depuis sa mise en place (masse volumique comprise entre 1400 et $1450 \mathrm{~kg} \mathrm{~m}^{-3}$ ). Deux sites de mesure comprenant 5 et 4 capteurs capacitifs respectivement ( 9 capteurs disponibles) sont mis en place (profondeurs 0,$025 ; 0,055 ; 0,085,0,125,0,175 \mathrm{~m}$, implantation horizontale pour les 2 premières profondeurs, implantation verticale pour les autres), ainsi que 2 tubes d'accès de $0,20 \mathrm{~m}$ de longueur pour les mesures de masse volumique par transmission gamma (Bertuzzi et al, 1987). Par ailleurs, des mesures de températures sont effectuées au centre de la case iysimétrique à l'aide de 5 sondes de platine préalablement étalonnées, disposées aux mêmes profondeurs que les capteurs capacitifs. Des contrôles destructifs d'humidité gravimétrique sont réalisés au cours du temps sur des placettes d'observation de petite dimension $(0,10 \mathrm{~m} \times 0,10 \mathrm{~m})$ réparties sur une grille carrée régulière. Les échantillons de sol sont prélevés à l'aide d'une petite tarière peu perturbatrice conçue à cet effet, et correspondent à des épaisseurs de sol fixées $(0-0,01 ; 0,01-0,02 ; 0,02-0,03$; $0,03-0,04 ; 0,04-0,05 ; 0,05-0,06 ; 0,06-0,07 ; 0,07-$ $0,10 ; 0,10-0,15 ; 0,15-0,20 \mathrm{~m})$. Une irrigation initiale par submersion $(80 \mathrm{~mm})$ est réalisée sur la surface du sol, et celle-ci est ensuite soumise à évaporation de façon continue (seule une pluie intervient en fin d'expérimentation). Par la suite, deux séries de mesures journalières sont réalisées (sondes capacitives, température, et humidité gravimétrique), l'une à $7 \mathrm{~h}$ le matin, l'autre à $14 \mathrm{~h}$. Pour chaque mesure capacitive réalisée le matin, on dispose de 3 contrôles gravimétriques de l'humidité répartis au hasard sur le site de mesure, et de 5 contrôles gravimétriques pour les mesures capacitives effectuées l'après-midi. Cette double mesure au cours de la journée (matin et après-midi) permet de trouver des situations où une faible variation de teneur en eau au cours de la journée coexiste avec une forte variation de température. 


\section{Expérience 2}

Contrairement à l'expérimentation précédente, cet essai se déroule sur une parcelle de grande dimension $(0,5 \mathrm{ha})$ de texture argilo-limoneuse, située sur le centre INRA de Montfavet (France), dans la plaine alluviale de la basse vallée de la Durance. Le sol est identique au sol intitulé Poirson dans l'étude en laboratoire (tableau 1). Cette parcelle sous culture de soja (variété Weber) dispose d'un système d'irrigation par aspersion. Globalement, l'état structural des horizons superficiels du sol est plus hétérogène que dans l'expérimentation précédente. Cette parcelle expérimentale est subdivisée en 5 sous-parcelles $(0,1$ ha), traitées selon le même itinéaire technique. L'une de ces sous-parcelles est utilisée pour l'implantation des sondes capacitives, des sondes de platine utilisées pour les contrôles de température, et des tubes d'accès permettant les mesures des profils de masse volumique par transmission gamma. Deux autres, situées de part et d'autre de la précédente, sont utilisées pour des contrôles gravimétriques destructifs de la teneur en eau. Quatre sites, comprenant chacun deux sondes capacitives implantées aux profondeurs 0,05 $\mathrm{m}$ et $0,10 \mathrm{~m}$, sont mis en place. Les sondes sont implantées verticalement dans le sol, entre 2 rangs de soja espacés de $30 \mathrm{~cm}$. Quatre profils de densité à proximité des sondes capacitives (de 0,02 à $0,20 \mathrm{~m}$, avec un pas de $0,02 \mathrm{~m}$ ) sont réalisés en début de campagne. Deux autres sites sont dédiés à l'obtention des profils thermiques $(0,02 \mathrm{~m} ; 0,05 \mathrm{~m} ; 0,10 \mathrm{~m}$; $0,15 \mathrm{~m} ; 0,20 \mathrm{~m}$ ). Les températures sont enregistrées toutes les $15 \mathrm{~min}$ sur une centrale d'acquisition de données (type SAM 60, constructeur AOIP, Evry, France) pendant la durée des mesures capacitives. En même temps que les mesures capacitives et thermiques, 8 profils gravimétriques ont été réalisés chaque jour, à l'aide de petites tarières $(0,00-0,01 ; 0,01-0,02$; $0,02-0,03 ; 0,03-0,04 ; 0,04-0,05 ; 0,05-0,07 ; 0,07-$ $0,10 ; 0,10-0,15 ; 0,15-0,20 \mathrm{~m})$. Les mesures capacitives et thermiques sont effectuées une fois par jour, le matin. Les capteurs capacitifs ont été préalablement étalonnés (conversion des fréquences en permittivité) en laboratoire en début de campagne. L'ensemble des mesures a débuté $16 \mathrm{j}$ après le semis et pendant une période de 40 j pendant la phase de croissance de la culture de soja, au cours de laquelle la parcelle a reçu 3 irrigations $(77,5 ; 57,5 ; 45,5 \mathrm{~mm}$ respectivement).

\section{RÉSULTATS ET DISCUSSION}

\section{Réponse du circuit électrique aux variations thermiques}

Les résultats sont donnés sur la figure 3 . Deux constatations peuvent être faites : d'une part, lorsque la sonde capacitive et l'air ambiant sont portés à des températures croissantes, il n'y a

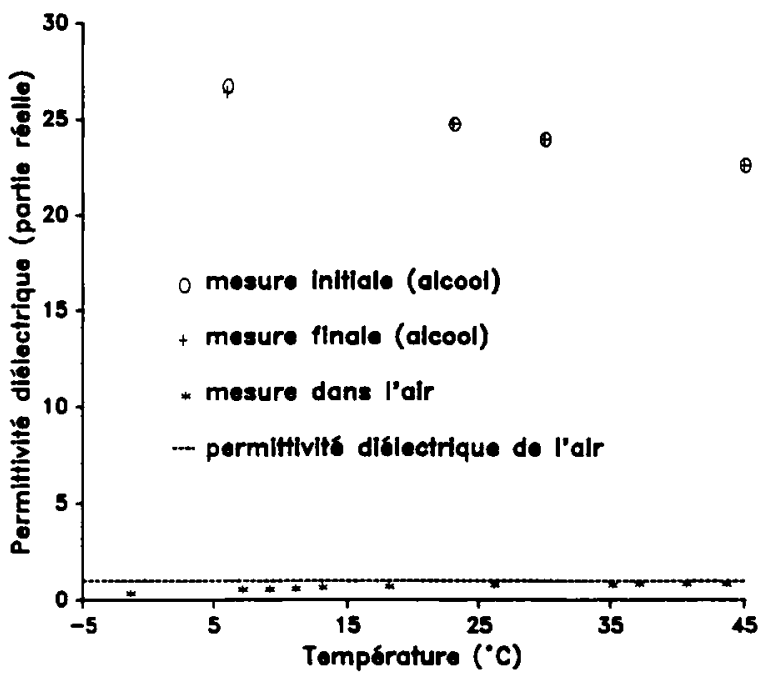

Fig 3. Effet de la température de la sonde capacitive sur sa réponse (air ou alcool). 0 : la sonde capacitive est dans l'alcool et à $20^{\circ} \mathrm{C} ;+$ : la sonde capacitive est dans l'alcool et à la température de l'alcool; * : la sonde capacitive est dans l'air et à la température de l'air.

pas d'évolution significative de la permittivité diélectrique relative mesurée. D'autre part, lorsque la sonde est plongée dans l'alcool pour 2 températures différentes du capteur $\left(20^{\circ} \mathrm{C}\right.$, et température de l'alcool), on n'observe pas non plus de différence significative entre les 2 mesures successives. Au total, les variations de permittivité diélectrique relative pouvant être imputées à des variations d'origine thermique du capteur sont faibles ou négligeables, toujours inférieures à 0,5 . Autrement dit, le dispositif choisi de double mesure alternée peut être considéré comme une solution efficace vis-à-vis de la stabilité thermique du capteur. Ces résultats sont similaires à ceux présentés par Dean et al (1987), qui sont les seuls à proposer un capteur également muni d'un dispositif électronique de limitation des dérives thermiques.

\section{Description de la géométrie du volume de mesure}

La figure 4 montre les effets d'un enfoncement vertical de la sonde capacitive dans l'alcool sur la valeur de permittivité relative obtenue. Pour un enfoncement de $27 \mathrm{~mm}$ (longueur de l'électrode en pointe), la mesure de permittivité relative correspond à $60 \%$ de la valeur théorique de la permittivité relative de l'alcool. Lorsque l'électrode annulaire est progressivement immergée dans le milieu, on observe une variation brutale 


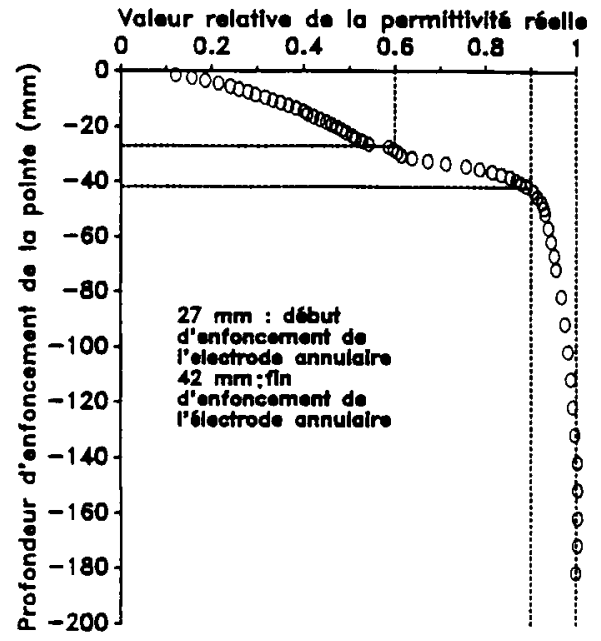

Fig 4. Effet de l'interface air (permittivité relative 1) alcool (permittivité relative 25) sur la mesure de permittivité diélectrique (l'ordonnée 0 correspond à l'extrémité de l'électrode en pointe.

de la réponse du capteur (fig 4), et il suffit alors d'un faible enfoncement supplémentaire $(15 \mathrm{~mm})$ pour que la mesure corresponde à $90 \%$ de la valeur théorique (enfoncement total de $42 \mathrm{~mm}$ ). Lorsque l'enfoncement atteint $100 \mathrm{~mm}$ environ, il n'y a pratiquement plus de variation de la permittivité relative mesurée (variation inférieure à $2,5 \%$ ). On constate donc une forte contribution sur la mesure de la zone située à proximité des électrodes, sur une longueur de quelques centimètres.

Ces résultats ne donnent évidemment qu'un ordre de grandeur des volumes de mesures existant pour un sol réel, pour plusieurs raisons. D'une part, ce volume de mesure dépend de la permittivité diélectrique relative du sol considéré. L'alcool, ayant une permittivité diélectrique relative $\varepsilon^{\prime}$ égale à 25 , correspond approximativement au cas d'un sol humide. D'autre part, ce volume de mesure peut aussi dépendre de la conductance électrique du milieu considéré. Dans le cas de l'alcool, les mesures effectuées indiquent des valeurs de conductance électrique de l'ordre de $1 \mathrm{mS}$. Cette valeur de conductance électrique, bien que très compatible avec celles rencontrées in situ dans nos propres expériences (1 à $4 \mathrm{mS}$ ), ne peut représenter à elle seule toute la gamme des valeurs possibles pour les sols réels. Enfin, l'alcool correspond à un milieu homogène et continu, ce qui n'est pas le cas d'un sol réel à structure généralement agrégée, hétérogène et discontinue. Dans la littérature, peu d'indications sont fournies sur l'ordre de grandeur des volumes de mesure pour les différents capteurs capacitifs existants. Cependant, des volumes de mesure de quelques dizaines de $\mathrm{cm}^{3}$ sont généralement avancés lorsque des indications existent à ce sujet (Kuraz, 1981; Bell et al, 1987; Dean et al, 1987).

\section{Effet du contact sol-électrodes}

Les résultats de cette expérimentation sont donnés par la figure 5 . Quelle que soit la nature du milieu initial situé au contact des électrodes (alcool ou air), la mise en place d'une couche de paraffine recouvrant les électrodes perturbe la mesure de permittivité relative. Dans le cas de l'alcool, une couche continue de paraffine de 0,4 $\mathrm{mm}$ induit une réduction importante de la valeur de permittivité diélectrique relative mesurée (de 27 à 13 environ). Pour une épaisseur de paraffine de 4 à $5 \mathrm{~mm}$, les valeurs de permittivité relative mesurées correspondent à des valeurs proches de la permittivité relative de la paraffine $(2,7)$. La qualité du contact sol-électrodes est donc probablement un facteur important de la qualité de la mesure, et la zone de mesure à proximité immédiate des électrodes semble jouer un grand rôle. Cela n'est pas sans conséquences sur le choix des modalités d'implantation du capteur in situ. Malgré tout, il faut relativiser ces résultats dans la mesure où nous avons pris en compte des situations extrêmes (capteur totalement isolé du sol par la paraffine). L'effet du contact sol-capteur, malgré son importance, est souvent ignoré dans les études des performances de capteur capacitif. À notre connaissance, seuls Dean et al (1987) fournissent des résultats à ce sujet, tout à fait similaires à nos propres observations.

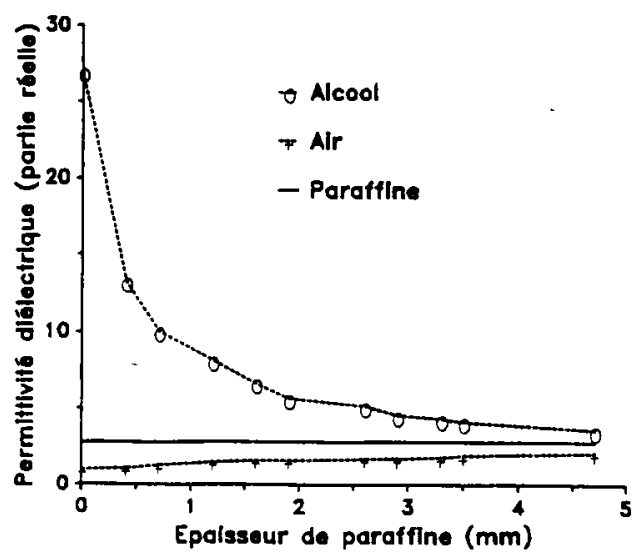

Fig 5. Effet d'une couche de paraffine autour des électrodes sur la mesure de permittivité diélectrique relative (air et alcool). 


\section{Relations d'étalonnage permittivité relative-teneur en eau volumique, obtenues en laboratoire}

\section{Effets de la texture}

Les résultats obtenus pour 3 matériaux choisis dans le cas d'une structure continue sont donnés sur la figure 6 . En premier lieu, on observe que, quel que soit le sol, la relation entre la permittivité diélectrique relative et la teneur en eau volumique est linéaire sur toutes les gammes de teneur en eau testées : les coefficients de corrélation linéaire sont toujours supérieurs à 0,99 et les écarts types résiduels sont compris entre 0,1 et 0,4 . Les pentes des relations expérimentales entre la permittivité relative et la teneur en eau volumique sont comparables entre elles $(54,6$; 54,7 et $61,3 \mathrm{~m}^{-3} \mathrm{~m}^{3}$ pour les matériaux $\mathrm{S} 31$, Flassan et Marçon respectivement). Les ordonnées à l'origine (permittivité relative à l'état $\mathrm{sec}$ ) sont égales à $4,1,9,2$ et 6,6 pour ces mêmes matériaux, et sont proches des valeurs attendues de permittivité à l'état $\sec (2$ à 7$)$.

La linéarité des relations obtenues peut sembler peu conforme aux estimations de permittivité diélectrique issues de modèles de mélanges, qui prévoient une relation non linéaire aux faibles humidités. D'un point de vue théorique, cette nonlinéarité des relations permittivité-teneur en eau prévue par les modèles de mélange aux faibles teneurs en eau est très dépendante de la présence d'eau liée électriquement à la matrice solide. Dans notre cas, les teneurs en argile de nos sols relativement faibles d'une part, l'absence de

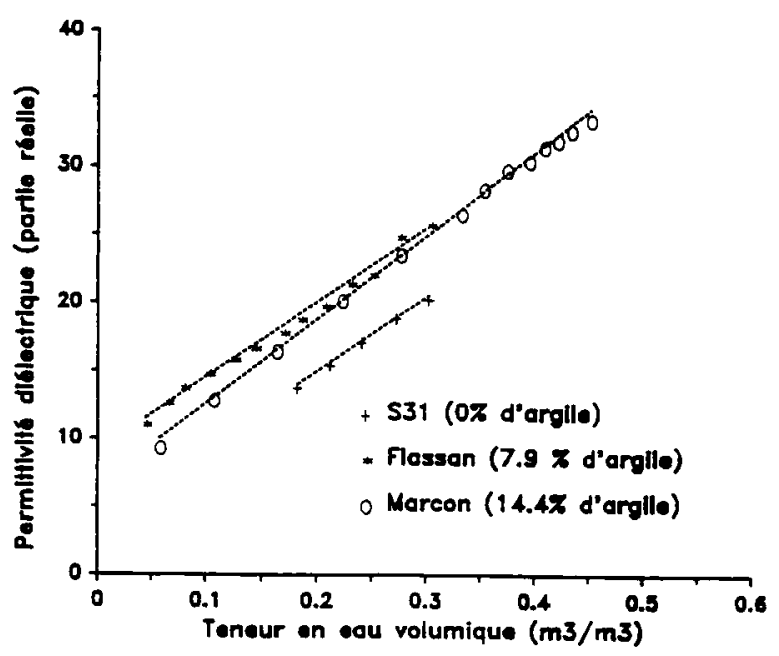

Fig 6. Relations permittivité diélectrique relative mesuréeteneur en eau volumique pour 3 matériaux à structure continue. mesure disponible à des teneurs en eau inférieures à $0,05 \mathrm{~m}^{3} \mathrm{~m}^{-3}$ d'autre part, peuvent expliquer l'absence d'un domaine non linéaire. Par ailleurs, une grande hétérogénéité existe dans la littérature à propos du caractère linéaire ou non des relations expérimentales permittivité relative-teneur en eau ou capacité électriqueteneur en eau : certains auteurs présentent des relations strictement linéaires sur une gamme plus ou moins large de teneur en eau (Paquet, 1965; Ambrosino, 1972; Manière et al, 1974; Saxena et Tayal, 1981), d'autres définissent des relations faiblement non linéaires pour certains matériaux, ou séparent des domaines de teneurs en eau correspondant à la juxtaposition de segments de droite (Paquet, 1965; Tran Ngoc Lan et al, 1970; Tran Ngoc Lan et al, 1972; Tran Ngoc Lan et Jallet, 1974; Bell et al, 1987: Campbell, 1990), d'autres enfin présentent des relations non linéaires dans tout le domaine de teneur en eau exploré (Wobschall, 1978; Kuraz, 1981). Cette diversité des réponses expérimentale reste de plus difficile à interpréter finement, dans la mesure où elle dépend à la fois des matériaux étudiés et de la gamme de teneur en eau explorée.

\section{Effets de la structure}

Les résultats relatifs à l'effet de la structure sur les relations permittivité relative-teneur en eau sont donnés sur la figure 7 . On constate d'une part un effet très important de la structure à proximité des électrodes sur les relations permittivité relative-teneur en eau volumique lorsqu'on passe d'une structure continue à une structure agrégée, d'autre part, très peu de différences

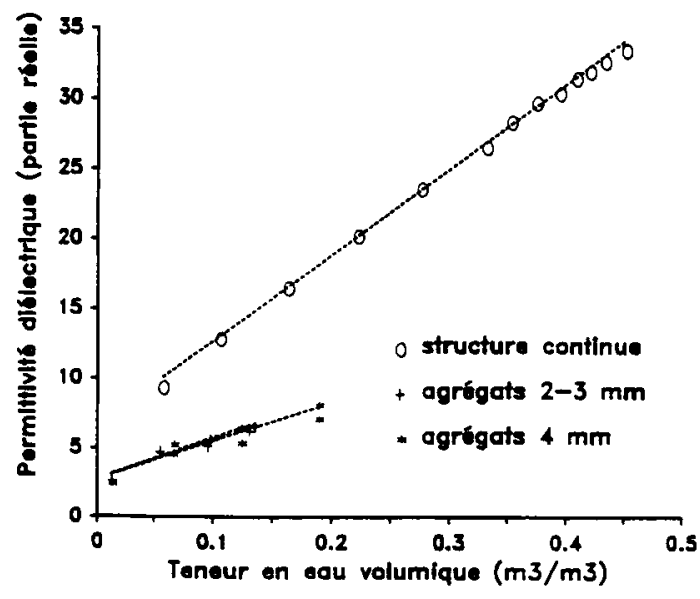

Fig 7. Effet de l'état structural sur les relations permittivité diélectrique relative-teneur en eau volumique. 
entre les structures obtenues, avec des agrégats de 2-3 $\mathrm{mm}$ ou de $4 \mathrm{~mm}$. Ce dernier point n'est d'ailleurs pas surprenant, compte tenu de la faible différence dans les tailles des agrégats choisies. La pente de la relation linéaire permittivité relative-teneur en eau passe de $61,3 \mathrm{~m}^{-3}$ $\mathrm{m}^{3}$ pour la structure continue, à 30,0 et $27,4 \mathrm{~m}^{-3}$ $\mathrm{m}^{3}$ pour les structures réalisées avec les agrégats de 2-3 $\mathrm{mm}$ et $4 \mathrm{~mm}$ respectivement. De même, l'ordonnée à l'origine qui mesure la permittivité relative du mélange de la matrice solide et de l'air, passe de 6,6 pour la structure continue à 2,8 pour les autres structures. Cette réduction de l'ordonnée à l'origine et cette diminution de la sensibilité de la réponse en permittivité relative pour les structures agrégées sont dues à l'important volume d'air situé à proximité immédiate des électrodes (permittivité relative de l'air égale à 1) et dont nous avons vu l'importance. Ces résultats soulignent l'effet primordial de l'arrangement géométrique des agrégats à proximité du capteur et donc, du soin qui doit être apporté lors de l'implantation de la sonde. Malgré tout, ces résultats doivent être relativisés. En effet, dans le cas des structures agrégées étudiées, la sonde n'est pas implantée dans le matériau pulvérulent, mais ce sont les agrégats qui sont disposés manuellement autour du capteur : chaque agrégat correspondant approximativement à une sphère n'est donc en contact avec les électrodes que par un seul point, et il est alors probable que la masse volumique locale à proximité immédiate de la sonde est en réalité plus faible que ne l'indique la mesure globale de masse volumique de l'échantillon. Les résultats de la bibliographie relatifs à ces effets de la structure sont peu nombreux, contradictoires, et difficiles à comparer entre eux. Ainsi, certains auteurs ne mettent pas en évidence d'effet de la densité (Manière et al, 1974; Kuraz, 1981). En revanche, d'autres mettent en évidence des effets (Ambrosino, 1972), ou montrent que l'introduction d'une nouvelle variable définie par le rapport capacité électrique / densité permet d'obtenir des relations indépendantes de la densité (Tran Ngoc Lan et al, 1970; Tran Ngoc Lan et al, 1972).

\section{Effets de la température}

Les résultats relatifs aux effets de la température sur les relations permittivité diélectrique relativeteneur en eau sont donnés sur la figure 8 . On observe un effet important de la température (de 0 à $45^{\circ} \mathrm{C}$ ) sur la permittivité relative, quelle que

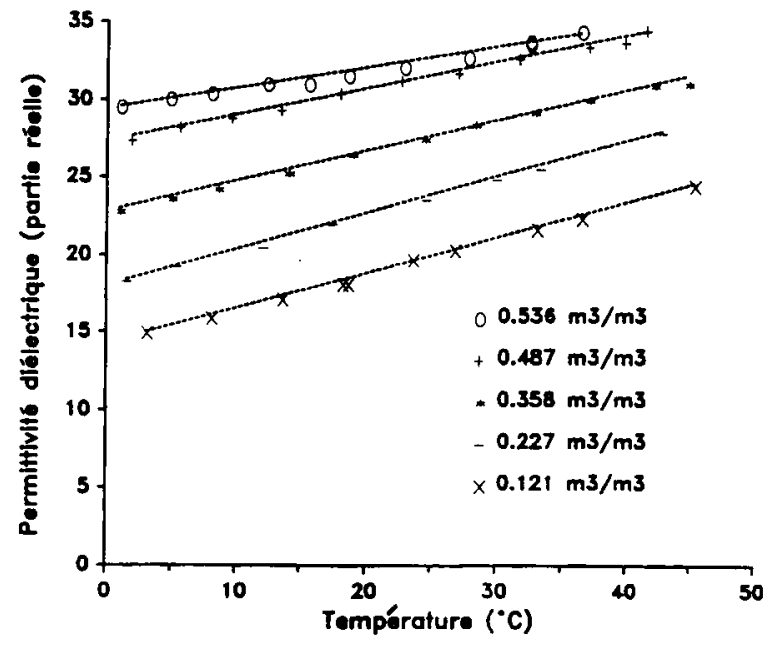

Fig 8. Relations entre la permittivité diélectrique relative mesurée et la température pour différentes teneurs en eau volumique (matériau Poirson).

soit la teneur en eau volumique (de 0,12 à 0,54 $\left.\mathrm{m}^{3} \mathrm{~m}^{-3}\right)$. Ces variations de permittivité relative liées aux variations de température ne peuvent être imputées au fonctionnement du capteur luimême, les tests de stabilité thermique de l'électronique ayant levé toute ambiguïté à cet égard. Les valeurs de pentes [d $\left.\varepsilon^{\prime} / \mathrm{d} T\right]$ observées pour chaque teneur en eau volumique choisie sont de $0,228,0,233,0,197,0,170$ et $0,132{ }^{\circ} \mathrm{C}^{-1}$ pour des teneurs en eau volumiques de $0,121,0,227$, $0,358,0,487,0,536 \mathrm{~m}^{3} \mathrm{~m}^{-3}$, respectivement. La sensibilité de la permittivité diélectrique relative à la température croît lorsque la teneur en eau volumique de l'échantillon diminue. Dans une assez large gamme de teneur en eau volumique éloignée de la saturation $\left(0,12\right.$ à $0,36 \mathrm{~m}^{3} \mathrm{~m}^{-3}$ dans notre cas), la valeur de la pente $\left[\mathrm{d} \varepsilon^{1 / d} \mathrm{dT}\right.$ ] reste assez stable $(0,20$ à 0,23$)$. Dans le cas des sols humides, l'influence de la température sur la permittivité diélectrique relative des mélanges solide-eau est assez complexe : en effet, à l'état libre, les liquides voient leur permittivité relative décroître avec la température, alors que c'est l'inverse pour les solides. Dans le cas des mélanges, cette augmentation de permittivité relative avec la température, déjà constatée par Hoekstra et Delaney (1974), serait liée aux propriétés diélectriques particulières de l'eau à l'état liè. Ces relations entre permittivité relative mesurée et température sont peu étudiées dans la littérature, et on ne distingue pas toujours clairement les effets réels, de ceux éventuellement liés à la dérive thermique du capteur. Ainsi, 
Kuraz (1981) met en évidence des effets analogues aux nôtres, mais de moindre amplitude, tandis que Campbell (1990) met en évidence des effets thermiques de sens opposé. Au vu de nos propres résultats, nous pensons que les effets thermiques sur la permittivité diélectrique relative des sols doivent impérativement être pris en compte et mériteraient une analyse exhaustive.

\section{Effets de la salinité}

La figure 9 présente les résultats obtenus en ce qui concerne l'effet de la salinité d'un sable pur saturé par une solution sur la permittivité diélectrique relative mesurée. On constate que jusqu'à des normalités d'environ $10^{-2}$, la permittivité relative mesurée est insensible à la concentration de la solution saline (conductance électrique de l'ordre de 2 à $3 \mathrm{mS}$ ), et reste égale à la permittivité relative du sable saturé avec de l'eau distillée. En revanche, on observe une agumentation rapide de permittivité relative pour des concentrations plus fortes. L'existence de ce seuil observé tendrait ainsi à démontrer l'efficacité du dispositif de compensation de la composante conductive du système sol-électrodes, uniquement pour des conductances inférieures à 2 à $3 \mathrm{mS}$. Ces effets liés à la salinité ne sont pas sytématiquement étudiés dans la littérature. Kuraz (1981) met en évidence des effets similaires aux nôtres $(0,1 \mathrm{~N}$ et $0,2 N)$, et Tran Ngoc Lan et al $(1970,1972)$ ne mettent en évidence aucun effet pour des concentrations allant de $0,5 \mathrm{~g} \mathrm{I}^{-1}$ à $2 \mathrm{~g} \mathrm{l}^{-1}(\mathrm{NaCl}$, $\left.\mathrm{CaSO}_{4}, \mathrm{Ca}(\mathrm{OH})^{2}\right)$.

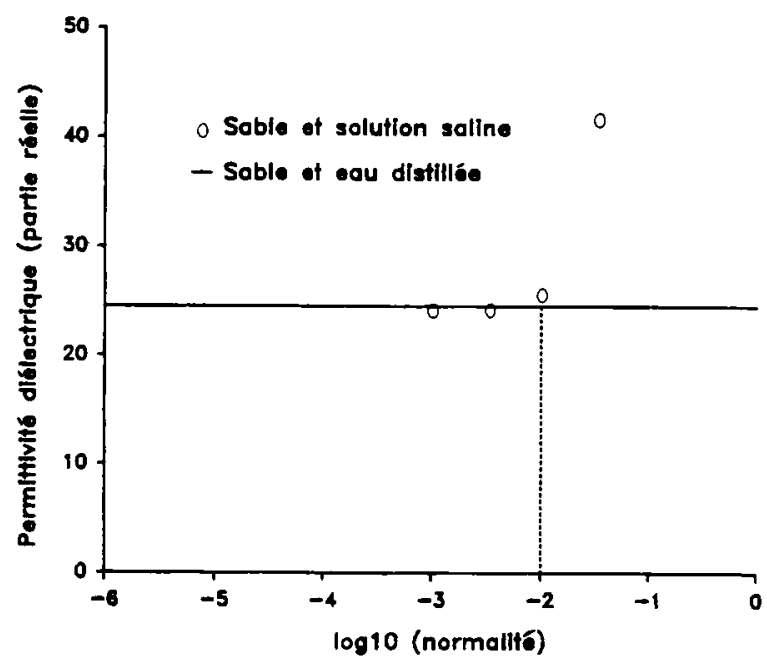

Fig 9. Relation entre la permittivité diélectrique relative mesurée et la concentration d'une solution saline saturant un sable pur.

\section{Expérimentation in situ}

\section{Données globables}

Des exemples de l'évolution simultanée des grandeurs physiques mesurées (permittivité diélectrique relative brute non corrigée des effets thermiques, teneur en eau volumique, température) sont donnés sur les figures 10a, b, c (expérience 1) et 11a, b, c (expérience 2). Globalement, on constate que les évolutions des teneurs en eau dans le sol et des valeurs de permittivité diélectrique mesurées ont des allures similaires. Pour chaque expérience, on observe un classement cohérent des capteurs capacitifs selon les profondeurs (faible permittivité diélectrique près de la surface plus sèche, forte permittivité en profondeur plus humide).

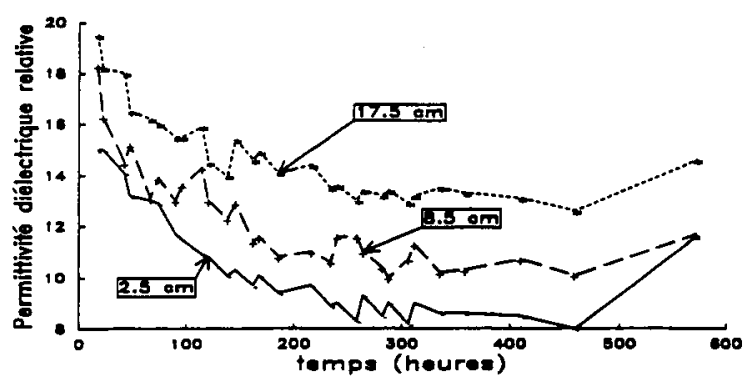

(a)

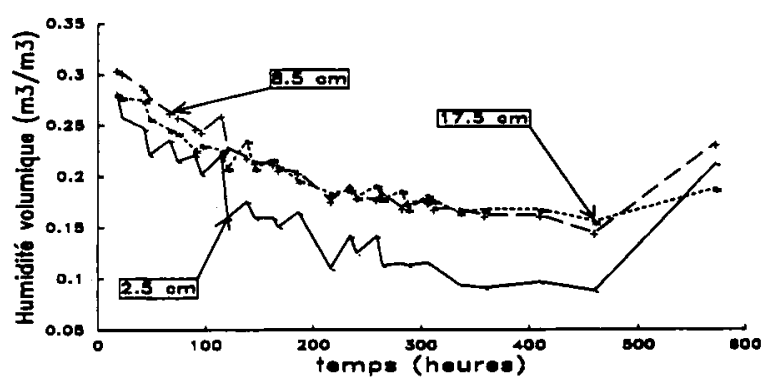

(b)

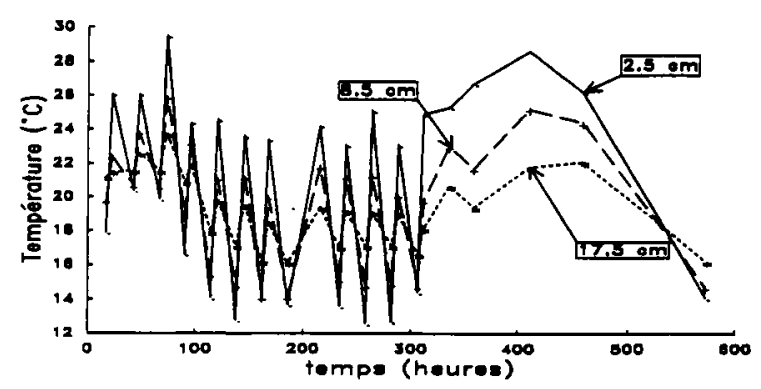

(c)

Fig 10. Évolution de la permittivité diélectrique relative, de la teneur en eau volumique et de la température du sol in situ (expérience 1) : a) permittivité diélectrique relative; b) teneur en eau volumique du sol; c) température du sol. 


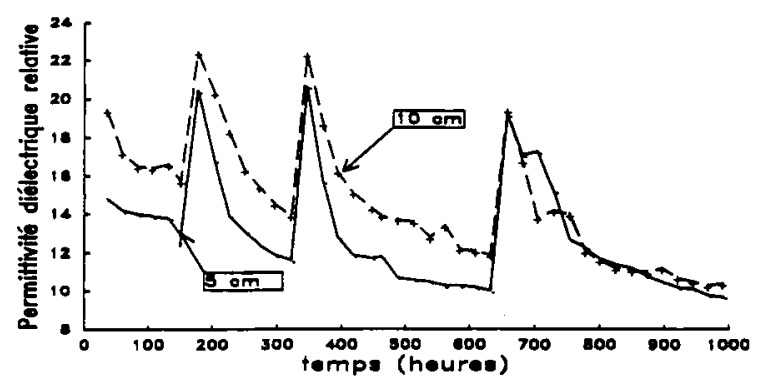

(a)

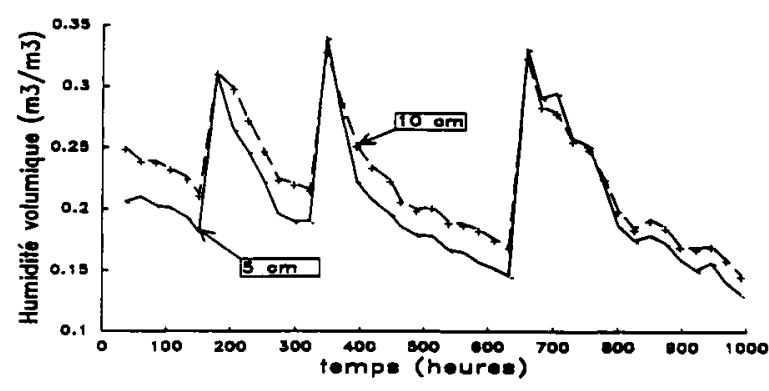

(b)

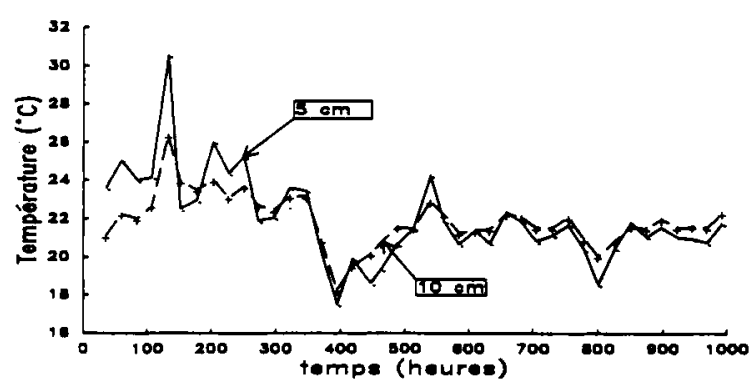

(c)

Fig 11. Évolution de la permittivité diélectrique relative, de la teneur en eau volumique et de la température du sol in situ (expérience 2) : a) permittivité diélectrique relative; b) teneur en eau volumique du sol; c) température du sol.

La teneur en eau volumique apparaît bien comme le premier facteur déterminant la réponse capacitive, indépendamment des autres sources possibles de variation. Par ailleurs, des fluctuations "en dents de scie» de la réponse capacitive apparaissent sur les résultats de l'expérience 1 (fig 10a). L'analyse détaillée de ces dents de scie montre qu'elles correspondent à des alternances entre des mesures du matin et de l'après-midi. La valeur de permittivité diélectrique mesurée le matin est en général plus élevée que celle obtenue en début d'après-midi, et ces variations sont en phase avec les variations d'humidité mesurées dans le sol. D'un point de vue physique, ces variations apparaissent cohé- rentes puisque la teneur en eau est plus élevée le matin qu'en début d'après-midi au maximum de l'évaporation, et il doit donc en être de même pour la permittivité diélectrique relative. Cependant, entre le matin et le midi, parallèlement au dessèchement du sol, on observe un échauffement des horizons superficiels qui peut atteindre une dizaine de degrés (fig 10c), et qui tend à augmenter les valeurs de permittivité mesurées. Ainsi, dans une telle situation expérimentale, on observe l'existence de deux sources de variation simultanées et de sens contraire sur la réponse capacitive, et il est donc nécessaire de séparer ces deux effets sur la mesure capacitive par une analyse plus détaillée de ces facteurs sur les relations d'étalonnage.

\section{Relations d'étalonnage}

Les figures 12 et 13 présentent des exemples de relations d'étalonnage permittivité diélectrique (non corrigée de la température)-teneur en eau volumique obtenues sur quelques capteurs représentatifs de l'ensemble des réponses capacitives obtenues au cours des expérimentations. Dans le cas de l'expérience 1 (tableau II), on retrouve pour chaque capteur des réponses linéaires comme en laboratoire ( $r^{2}$ compris entre 0,78 et 0,91 , pentes comprises entre 32,0 et 64,9 $\left.\mathrm{m}^{-3} \mathrm{~m}^{3}\right)$. On observe des écarts type résiduels qui, bien que normalement supérieurs à ceux observés en laboratoire $(0,1$ à 0,4$)$ restent faibles $\left(0,6\right.$ à 1,2 soit 0,02 à $0,03 \mathrm{~m}^{3} \mathrm{~m}^{-3}$ en teneur en eau). Les mêmes constatations globales sont applicables au cas de l'expérience 2 (fig 12, $\mathrm{r}^{2} \mathrm{com}$ pris entre 0,78 et 0,90 mise à part une valeur égale à 0,55 , pentes comprises entre 44,0 et $62,8 \mathrm{~m}^{-3} \mathrm{~m}^{3}$ ).

Les pentes obtenues pour tous les capteurs sur l'ensemble des deux expériences appartiennent à la gamme $\left(32,0-64,9 \mathrm{~m}^{-3} \mathrm{~m}^{3}\right)$ et sont compatibles avec les ordres de grandeurs obtenus en laboratoire pour différents sols et différents états structuraux $\left[27,4-61,3 \mathrm{~m}^{-3} \mathrm{~m}^{3}\right]$. Par ailleurs, quelle que soit l'expérience considérée, on observe des ordres de grandeur des ordonnées à l'origine (permittivité du milieu à l'état sec) tout à fait cohérents $(1,8 ; 1,9 ; 3,5 ; 4,8 ; 5,3 ; 5,4$; 5,$4 ; 5,5 ; 8,9$ pour l'expérience 1 , et 0,$5 ; 0,5 ; 2,0$; 2,$1 ; 2,2 ; 3,7 ; 4,8 ; 8,0$ pour l'expérience 2 ).

La variabilité des relations d'étalonnage observée d'un site à l'autre peut être expliquée par plusieurs phénomènes. D'une part, la variabilité 

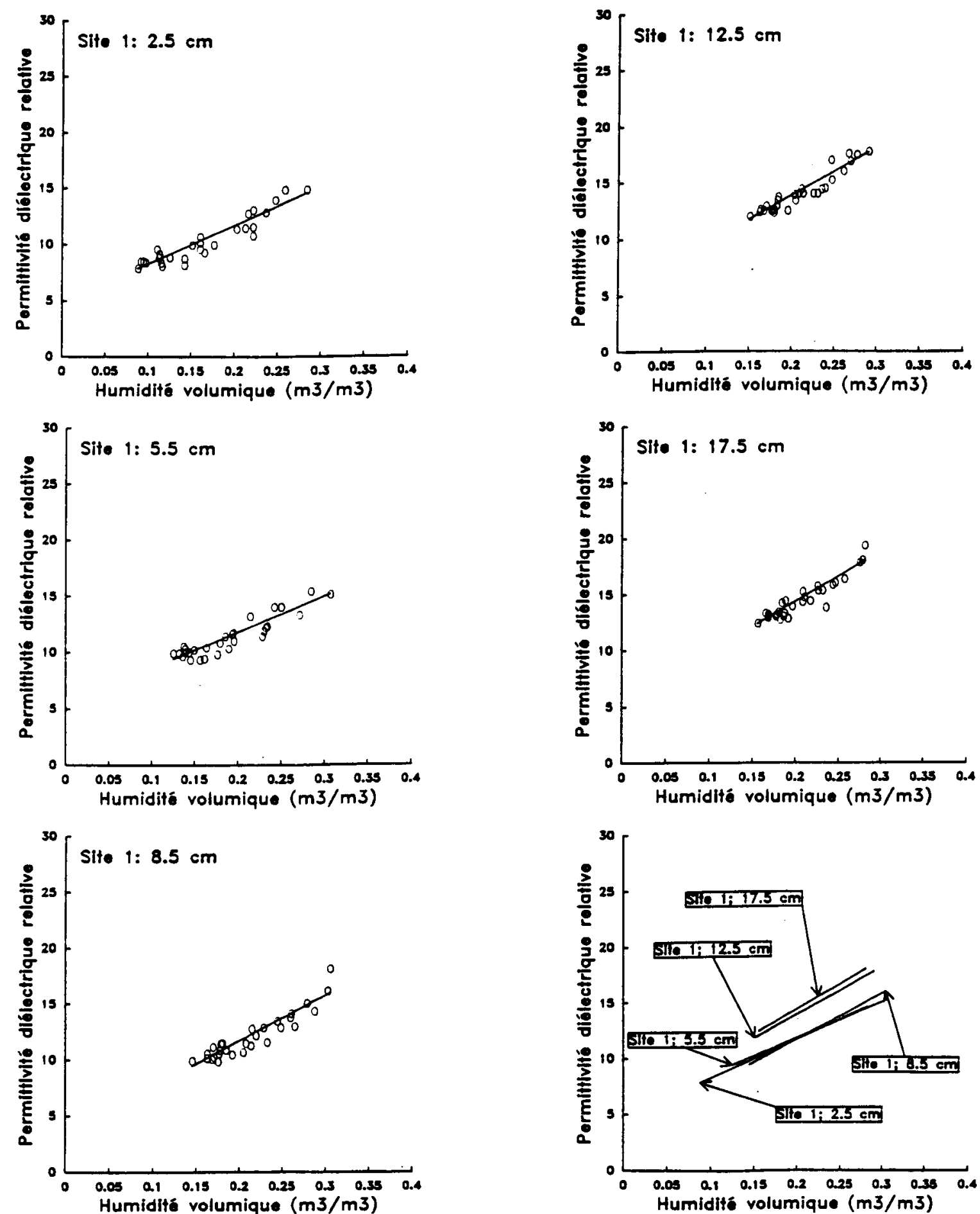

Fig 12. Exemple de relations d'étalonnage (permittivité diélectrique relative en fonction de la teneur en eau volumique) obtenues in situ pour différents capteurs (expérience 1).

spatiale de la teneur en eau d'un site à l'autre (certains sites étant plus humides que d'autres, et le restant systématiquement au cours du temps) peut rendre compte d'une partie des écarts systématiques observés d'un capteur à l'autre. Dans le cas de l'expérience 1, les écarts type obtenus sur les teneurs en eau mesurées dans le sol à l'échelle de la parcelle sont généralement de l'ordre de 0,01 à $0,02 \mathrm{~m}^{3} \mathrm{~m}^{-3}$, et dans le cas de l'expérience 2, ils sont de l'ordre de 0,01 à $0,03 \mathrm{~m}^{3} \mathrm{~m}^{-3}$ au maximum. Ces ordres de grandeur de la variabilité spatiale des teneurs en 

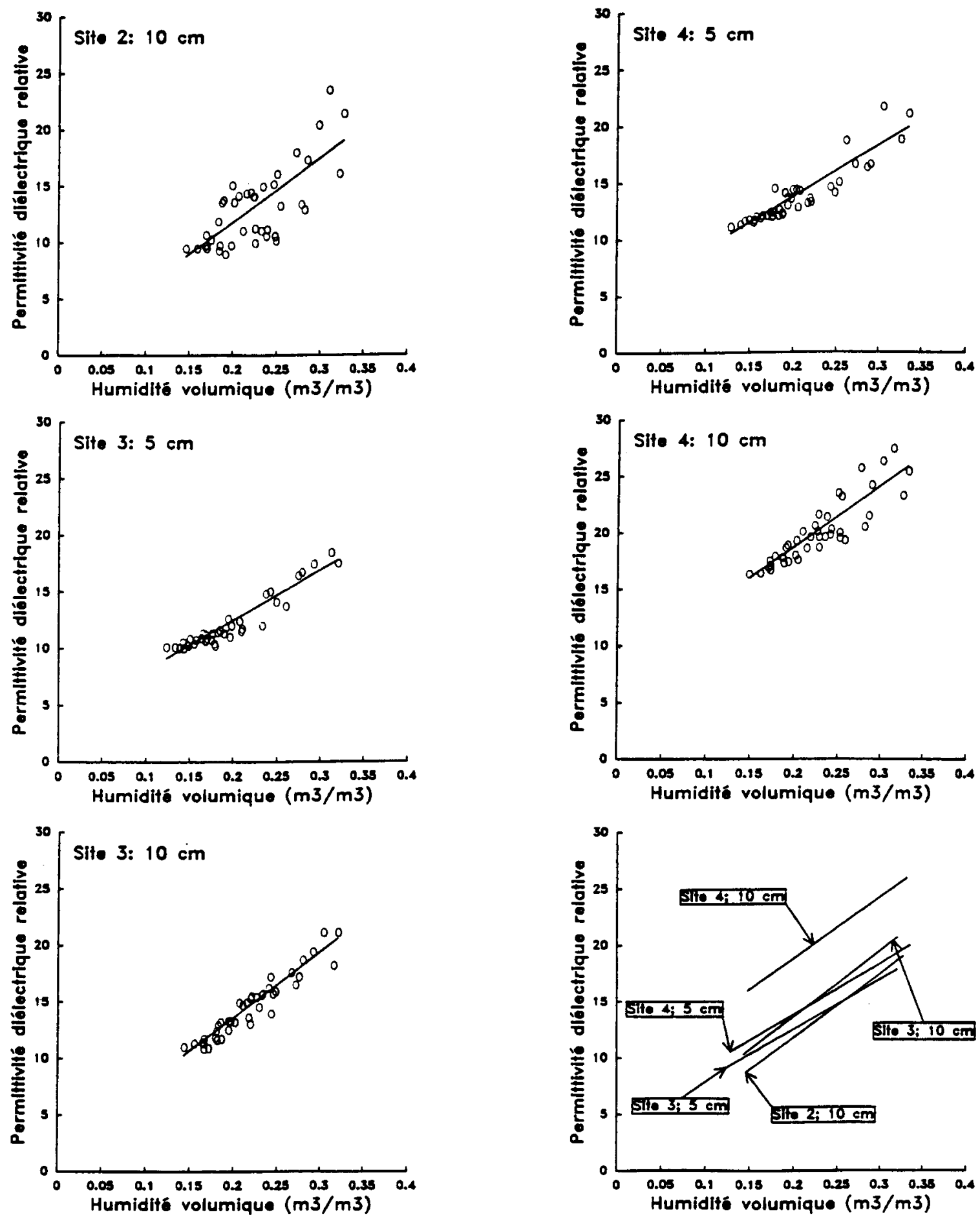

Fig 13. Exemple de relations d'étalonnage (permittivité diélectrique relative en fonction de la teneur en eau volumique) obtenues in situ pour différents capteurs (expérience 2).

eau du sol apparaissent relativement faibles, mais peuvent cependant expliquer une part des variations de permittivité diélectrique relative rencontrées d'un capteur à l'autre (une variation de teneur en eau volumique de $0,01 \mathrm{~m}^{3} \mathrm{~m}^{-3}$ correspond, à une variation de 0,5 pour la permittivité relative environ). D'autre part, la variabilité des réponses individuelles observée d'un capteur à l'autre peut également être liée à l'environnement immédiat du capteur, qu'il s'agisse de l'état structural ou du contact sol-électrodes résultant de son mode d'implantation. Les résultats obte- 
Tableau II. Principales caractéristiques statistiques des régressions linéaires simple (teneur en eau $\theta$ ) et multiple ( $\theta$ et T) pour l'expérience 1, en utilisant la permittivité diélectrique relative comme variable à expliquer.

\begin{tabular}{|c|c|c|c|c|c|c|c|c|c|}
\hline \multicolumn{2}{|c|}{ Capteurs } & \multicolumn{4}{|c|}{ Régression $\theta$} & \multicolumn{4}{|c|}{ Régression $\theta$ et $T$} \\
\hline Site & Prof & $\begin{array}{l}\text { Pente } \\
m^{-3} m^{3}\end{array}$ & Ordo & $r^{2}$ & $\sigma$ & $\begin{array}{l}\text { Pente } \theta \\
m^{-3} m^{3}\end{array}$ & $\begin{array}{l}\text { Pente T } \\
{ }^{\circ} \mathrm{C}-1\end{array}$ & $\mathrm{r}^{2}$ & $\sigma$ \\
\hline 1 & 2,5 & 34,4 & 4,8 & 0,88 & 0,74 & 36,4 & 0,11 & 0,96 & 0,43 \\
\hline 1 & 5,5 & 32,0 & 5,4 & 0,85 & 0,70 & 33,3 & 0,12 & 0,95 & 0,40 \\
\hline 1 & 8,5 & 40,8 & 3,5 & 0,86 & 0,77 & 40,3 & 0,07 & 0,87 & 0,74 \\
\hline 1 & 12,5 & 42,6 & 5,3 & 0,89 & 0,60 & 41,0 & 0,11 & 0,93 & 0,49 \\
\hline 1 & 17,5 & 44,8 & 5,4 & 0,87 & 0,65 & 42,4 & 0,10 & 0,88 & 0,63 \\
\hline 2 & 2,5 & 42,8 & 8,9 & 0,78 & 1,22 & 46,0 & 0,17 & 0,92 & 0,78 \\
\hline 2 & 5,5 & 44,2 & 5,5 & 0,87 & 0,83 & 45,7 & 0,12 & 0,94 & 0,57 \\
\hline 2 & 8,5 & 59,4 & 1,9 & 0,91 & 0,81 & 58,7 & 0,10 & 0,93 & 0,74 \\
\hline 2 & 12,5 & 64,9 & 1,8 & 0,90 & 0,85 & 61,9 & 0,22 & 0,96 & 0,54 \\
\hline
\end{tabular}

nus en laboratoire (fig 7) suggèrent du moins une telle hypothèse.

\section{Effets simultanés de la teneur en eau, de la température et de la conductance électrique sur la réponse capacitive in situ}

Pour mettre en évidence dans ces réponses globales des capteurs capacitifs les effets simultanés de la teneur en eau et de la température préalablement mis en évidence en laboratoire, nous avons traité les données par une régression linéaire multiple, en utilisant la teneur en eau volumique $(\theta)$ et la température $(T)$ comme variables explicatives, et la permittivité diélectrique relative $\left(\varepsilon^{\prime}\right)$ mesurée comme variable expliquée. Le modèle choisi est le suivant :

$$
\varepsilon^{\prime}=a \theta+b T+\xi
$$

Le choix de cette procédure statistique est déterminé par les résultats obtenus en laboratoire, qui montrent qu'à température constante, la permittivité relative présente des réponses linéaires visà-vis de l'humidité, et que, à teneur en eau volumique constante, il en est de même en ce qui concerne la réponse de la permittivité diélectrique relative vis-à-vis de la température. Cette procédure n'a été appliquée qu'à l'expérience 1, pour laquelle la double mesure quotidienne de température permet d'obtenir des contrastes thermiques importants pour de faibles variations de teneur en eau entre le matin et l'après-midi, et donc de bien mettre en évidence des effets ther- miques éventuels. L'introduction de la température est nette (tableau II), puisque la gamme des carrés des coefficients de corrélation linéaire passe de $(0,78-0,91$, cas de la régression simple) à $(0,87-0,96$, cas de la régression multiple), tandis que l'écart type résiduel après régression exprimé en permittivité relative passe de $(0,60-1,22)$ à $(0,40-0,78)$. De plus, on constate (tableau II) que l'introduction d'une seule variable $(\theta)$ ou de deux variables ( $\theta$ et $T$ ) ne modifie pas sensiblement la valeur du coefficient relatif à l'effet de la teneur en eau (coefficient «a» de l'équation [4]), ce qui confirme la pertinence d'un modèle additif sans interaction entre les variables (équation [4]). Les valeurs des coefficients de régression liés à la température $\left(0,070\right.$ à $0,217^{\circ} \mathrm{C}^{-1}$ dans le cas de l'expérience 1) sont du même ordre de grandeur que celles trouvées expérimentalement dans les expérimentations contrôlées en laboratoire $(0,13$ à $0,23^{\circ} \mathrm{C}^{-1}$ selon les teneurs en eau volumiques, dans le cas du sol de l'expérience 2). Enfin, on peut se demander si une partie de l'écart type résiduel après prise en compte des effets liés à la teneur en eau et à la température n'a pas pour origine les variations de conductance électrique au cours du temps. La réponse semble négative, puisque les fluctuations mesurées de conductance électrique dans le cas de l'expérience 2 (tableau III) sont limitées (coefficients de variation de 10 à $20 \%$ pour des valeurs moyennes de l'ordre de $2 \mathrm{mS}$ ), bien que le sol soit soumis à des alternances de phases d'humectation et de dessèchement. 
Tableau III. Valeurs minimales, maximales et moyennes des conductances électriques (mS) mesurées in situ dans le cas de l'expérience 2.

\begin{tabular}{lcccccccc}
\hline Site & 1 & 1 & 2 & 2 & 3 & 3 & 4 & 4 \\
Prof $(\mathrm{cm})$ & 5 & 10 & 5 & 10 & 5 & 10 & 5 & 10 \\
\hline$n$ & 39 & 40 & 40 & 39 & 39 & 41 & 40 & 40 \\
$\operatorname{Min}$ & 1,4 & 1,7 & 1,4 & 1,5 & 1,2 & 1,7 & 1,5 & 2,1 \\
$\operatorname{Max}$ & 3,0 & 2,8 & 2,4 & 2,8 & 2,0 & 2,8 & 2,4 & 4,0 \\
$m$ & 1,81 & 2,05 & 1,74 & 1,99 & 1,65 & 2,01 & 1,95 & 2,78 \\
$s$ & 0,32 & 0,25 & 0,22 & 0,35 & 0,24 & 0,23 & 0,21 & 0,59 \\
$\operatorname{CV}(\%)$ & 17,7 & 12,2 & 12,6 & 17,6 & 14,5 & 11,4 & 10,8 & 21,2 \\
\hline
\end{tabular}

\section{CONCLUSION}

Les différentes expérimentations présentées permettent d'évaluer globalement les qualités et les limites du capteur capacitif proposé, notamment dans une perspective d'utilisation sur le terrain. Les principaux points qui se dégagent sont les suivants :

- le capteur capacitif et l'électronique associée ne présentent pas de dérive thermique significative;

- le volume concerné par la mesure est essentiellement localisé à proximité des électrodes;

- pour une situation texturale donnée, 3 types de causes sont susceptibles de perturber la réponse capacitive vis-à-vis de la mesure de la teneur en eau : les variations de température, les variations de concentration saline et de conductance électrique associée, et enfin, l'ensemble des effets liés à l'état structural dans le voisinage immédiat des électrodes.

En ce qui concerne les effets liés à la température, ils peuvent être pris en compte par la conception-même du capteur, qui prévoit un dispositif de correction thermique. Pour ce qui concerne les effets liés à la salinité, le capteur capacitif apparaît capable de fonctionner sans perturbation, dans une gamme de situations dont il faudra préciser la probabilité d'existence. Dans le cas des effets liés à l'état structural du sol, les risques de mauvais contact sol-capteur apparaissent réels. Cela interdit d'envisager l'utilisation d'une relation d'étalonnage universelle, et un étalonnage in situ s'impose. Par ailleurs, ces risques incitent à placer les sondes capacitives dans le sol avec le plus grand soin. Par rapport aux capteurs capacitifs existants, les comparaisons restent difficiles à faire, compte tenu du caractère hé- térogène et souvent incomplet des études existantes. Le capteur proposé ouvre des perspectives de mesure de la teneur en eau qui peuvent être complémentaires par rapport aux autres techniques disponibles, et l'enregistrement automatique et éventuellement continu de mesures capacitives au cours du temps permettrait d'affiner nos conclusions, et probablement de mieux décrire des phénomènes mal appréhendés par manque de continuité des mesures (infiltration rapide, redistribution nocturne de l'eau dans les sols, prélèvements racinaires, par exemple).

\section{RÉFÉRENCES}

Ambrosino R (1972) Utilisation des oscillateurs pour les mesures capacitives de teneur en eau. Bull Lialson Labo P Ch 60, 166-172

Baron JP, Tran Ngoc Lan (1977) Méthodes de mesure et de contrôle des teneurs en eau des matériaux dans les laboratoires des ponts et chaussées. Bull Liaison Labo P Ch 87, 85-96

Bell JP, Dean TJ, Hodnett MG (1987) Soil moisture measurement by an improved capacitance technique, part II. Field techniques, evaluation and calibration. J Hydrol 93, 79-90

Bertuzzi P, Bruckler L, Gabilly Y, Gaudu JC (1987) Calibration, field-testing and error analysis of a gamma ray probe for in situ measurement of dry bulk density. Soil Sci 144, 425-436

Campbell JE (1990) Dielectric properties and influence of conductivity in soils at one to fifty Megahertz. Soil Sci Soc Am J 54, 332-341

Dean TJ, Bell JP, Baty JB (1987) Soil moisture measurement by an improved capacitance technique, part I. Sensor design and performance. J Hydrol 93, 67-78

Fumanal JC, Gaudu JC, Mathieu JM, Stengel P (1989) Sonde capacitive pour la mesure in situ de la teneur en eau d'un sol. Brevet Français 89.15135, 17 novembre 1989 
Hamid MA, Mostowy NJ (1976) Capacitive moisture probe for the prefab concrete industry. IEEE Trans Ind Electron Contr Instrum IECI-23 4, 462-465

Hoekstra P, Delaney A (1974) Dielectric properties of soil at UHF and microwave frequencies. $J$ Geographysical Res 79, 1699-1708

Kuraz V (1981) Testing a field dielectric soil moisture meter. Geotech Test J 4, 3, 111-116

Manière G, Luc JP, Baran R (1974) Utilisation des sondes capacitives en hydrologie. Bull Liaison Labo $P$ Ch $74,10-16$

Paquet J (1965) Application des méthodes électriques à la mesure de la teneur en eau des matériaux. Ann Inst Tech Bat Trav Publics 215, 1599-1610

Paquet J (1971) Mesure d'humidité in situ par les méthodes diélectriques, application au béton. Matér Construc 4, 20, 87-100

Saxena SC, Tayal GM (1981) Capacitive moisture meter. IEEE Trans Ind Electron Contr Instrum IECI28, 1, 37-39

Schmugge TJ, Jackson TJ, McKim HL (1980) Survey of methods for soil moisture determination. Water Resour Res 16, 961-979
Topp GC, Davis JL, Annan AP (1982) Electromagnetic determination of soil water content using TDR: I. Applications to wetting fronts and steep gradients. Soil Sci Soc Am J 46, 672-678

Tran Ngoc Lan, Chaigne P, Philippe A (1970) Mesure des teneurs en eau des sols par les méthodes électriques, étude d'une méthode capacitive. Laboratoire central des Ponts et Chaussées, section de géotechnique routière du département des sols, rapport de recherche numéro 5, $237 p$

Tran Ngoc Lan, Chaigne P, Philippe A (1972) Expérimentation d'une méthode capacitive pour l'évaluation de l'humidité des sols. Bull Liaison Labo P Ch 60, 155-165

Tran Ngoc Lan, Jallet A (1974) Mesure par capacité électrique de la teneur en eau des sables sur bande transporteuse. Bull Liaison Labo $P$ Ch 71, 109-115

Ulaby FT (1974) Radar measurement of soil moisture content. IEEE Trans Antennas Propag AP-22, 2, 257-265

Wobschall D (1978) A frequency shift dielectric soil moisture sensor. IEEE Trans Geosci Electron 16, $2,112-118$ 